\title{
ENDOCRINE RESPONSES TO PHYSICAL TRAINING AND TRIBULUS TERRESTRIS SUPPLEMEMTATION IN MIDDLE-AGE MEN
}

\author{
Michał Wilk, ${ }^{1, A, B, E}$ Małgorzata Michalczyk, 2, A, B, E Jakub Chycki,, E, B Adam Maszczyk, 1, B, C, D \\ Miłosz Czuba, ${ }^{1, E}$ Robert Roczniok, ${ }^{1, C, E}$ Artur Gołaś, ${ }^{1, B, E}$ Adam Zając ${ }^{1, A, E}$
}

\footnotetext{
1 Department of Sports Training, The Jerzy Kukuczka Academy of Physical Education, Katowice, Poland

2 Department of Sport Nutrition, The Jerzy Kukuczka Academy of Physical Education, Katowice, Poland

A Study Design; ${ }^{\text {B }}$ Data Collection; ${ }^{\mathrm{C}}$ Statistical Analysis; ${ }^{\mathrm{D}}$ Manuscript Preparation; ${ }^{\mathrm{E}}$ Funds Collection
}

\author{
Address for correspondence: \\ Adam Maszczyk Ph.D. Associate Professor \\ The Jerzy Kukuczka Academy of Physical Education \\ Department of Statistics and Methodology \\ Mikołowska 72a, 40-065 Katowice, Poland \\ E-Mail: a.maszczyk@awf.katowice.pl
}

\begin{abstract}
Ahstract. The aim of this study was to evaluate the effects of steroidal saponin supplementation on blood concentration of T, GH and IGF-1. The research involved 14 men between the age of 45 and 60 years. The duration of the experiment was 12 weeks. There were two series of laboratory tests. Independent tests were conducted at the beginning and after 12 weeks of the intervention.

A two-way repeated measures ANOVA revealed a statistically significant effect of the intervention on the following variables: T-Ch $\left(\eta^{2}=0.542\right), \mathrm{HDL}-\mathrm{Ch}\left(\eta^{2}=0.522\right)$, LDL-Ch $\left(\eta^{2}=0.587\right), \mathrm{T}\left(\eta^{2}=0.603\right)$, IGF-1 $\left(\eta^{2}=0.512\right)$ and GH $\left(\eta^{2}=0.621\right)$. Thus, FFM significantly increased while TBF and BM decreased in comparison to pre-intervention levels. The analyzed results indicate that treatment or supplementation of individual hormone deficiencies can be a successful form of counteracting the aging process. Nevertheless, the effects of TT supplementation on the concentration of T as well as GH and IGF-1, requires further studies, especially in middle-aged and older subjects, along with different exercise programs. The analyzed results indicate that treatment or supplementation of individual hormone deficiencies can be a major form of counteracting the aging process.
\end{abstract}

KeV WOrdls: steroidal saponins, testosterone, supplementation, growth hormone, insulin-like growth factor

\section{Introduction}

The neuroendocrine system plays a prominent role in controlling the rate of muscle tissue degradation and the efficiency of metabolic processes. Among the main morphological symptoms of aging the most obvious include: a decrease in lean body mass (LBM), an increase in body fat content (FM), disorders in lipid and lipoprotein profile, and a reduction in bone mineral density. The endocrine system, particularly anabolic hormones, such as growth hormone $(\mathrm{GH})$ and testosterone $(\mathrm{T})$, as well as the insulin-like growth factor (IGF-1) determine the anabolic processes occurring in the human body to a large extent. Hypogonadism and hiposomatropism are factors which 
directly contribute to a decrease in muscle mass. There are many conflicting results regarding the effects of supplementation on the concentration of particular anabolic and lypolytic hormones. The use of supplements before, during and after exercise has a significant impact on post-exercise blood hormone concentration (Bird, Tarpenning, Marino, 2006). The effect of changes in the concentration of various hormones, as a result of supplementation, is distinctly observed among older individuals, diagnosed with hormonal deficiencies.

Tribulus Terrestris (TT) is a supplement derived from the fenugreek plant. Specific properties of saponins, such as interaction with cell membranes, combining with sterols, as well as cholesterol, leads to partial damage and greater permeability. Steroidal saponins have a high capacity for erythrocyte hemolysis. By affecting the metabolism of male androgens saponins influence the increase of T concentration (Huang, Tan Jiang, Zhu, 2003). The exact mechanism of TT is not recognized, however, steroidal saponins inhibit the activity of key enzymes responsible for the unwanted conversion of testosterone to estrogen and dihydrotestosterone (DHT). Reduced production of unfavorable metabolites stimulates the secretion of testosterone by the body, and enhances its activity within the muscle tissue. Other researchers suggest that the ergogenic effect of saponins is primarily associated with the increase in the concentration of luteinizing hormone $(\mathrm{LH})$, which stimulates the secretion of testosterone (De Combarieu, Fizzati, Lovati, Mercalli, 2003). Gauthaman, Mohamed Saleem, Ravi, Sita, Niranjali (2008) showed that a TT dose of 900 to $2500 \mathrm{mg}$ per day stimulates the synthesis of $\mathrm{T}$ in rats. Despite theoretical basis and research on animals, which confirm that TT increases the concentration of $\mathrm{LH}$ and $\mathrm{T}$, the results of human studies are inconclusive. Studies in a group of healthy subjects with normal levels of $\mathrm{GH}$ and $\mathrm{T}$ do not confirm the impact of TT on anabolic functions (Rogerson et al., 2007).

Nonetheless, several studies have shown that TT, as well as vitamin and mineral supplementation does not increase muscle strength or muscle hypertrophy as a result of resistance training (Rogerson et al., 2010; Antonio, Uelmen, Rodriguez, Earnest, 2010). There were no significant changes in body composition and body mass; among men who were engaged in regular resistance exercise and TT supplementation (Antonio et al., 2010). A different situation occurs in case of TT supplementation among subjects with known hypogonadism and hiposomatropism. Adimoelja (2000) showed on a group of middle-aged subjects that TT has a significant impact on the concentration of $\mathrm{LH}$ and $\mathrm{T}$.

TT supplementation in subjects with known hypogonadism is also diabetogenic, prevents cancer, reduces total cholesterol (Ch-T) and low-density lipoprotein (LDL) (De Combarieu et al., 2003). Steroidal saponin supplementation in men with physiologically reduced concentrations of $\mathrm{T}$ and $\mathrm{GH}$ may be a reasonable alternative for hormone therapy, and as research indicates an increase in the concentration of $\mathrm{T}$ in patients with GHD or impaired secretion of testosterone significantly affects the changes of body composition and improves the physical abilities of the organism (Zając et al., 2010).

The aim of this study was to evaluate the effect of steroidal saponins supplementation on blood concentration of T, GH and IGF-1 and changes in body mass and body composition, as well as in the lipid and lipoprotein profile in middle-aged and slightly overweight men.

\section{Methods}

\section{Subjects}

The research involved 14 men between the age of $45-60$ years, body mass index of $25-33$, and body fat content between $23-30 \%$. The subjects were randomly divided into two groups - an experimental (exp) and 
a control group (contr). The exp. group received steroidal saponins; for the first six weeks three capsules (900 mg) per day in split doses. Two capsules were ingested in the morning on an empty stomach $(600 \mathrm{mg})$ and one at bedtime (300 mg). From weeks 6 to 12, 6 capsules $(1,800 \mathrm{mg}$ ) were ingested per day in split doses. Four capsules in the morning on an empty stomach $(1,200 \mathrm{mg})$ and two at bedtime $(600 \mathrm{mg})$. The contr. group received a placebo in the form of gelatin capsules. During the 12 weeks of the experiment all subjects participated in a physical activity program. Prior to testing, as well as in the course of the experiment, participants followed an isocaloric mixed diet (55\% carbohydrate, $20 \%$ protein, $25 \%$ fat).

\section{Research methods}

\section{Test procedure}

The duration of the experiment was 12 weeks. There were two series of laboratory tests. Independent tests were conducted at the beginning and after 12 weeks of the intervention. The study evaluated such morphological, physiological and biochemical variables as body mass and body composition (BM, FFM, FM, TBW, BMI), the concentration of chosen hormones and growth factors (T, GH, IGF-1), as well as the lipid and lipoprotein profile including: triglycerides (TAG), total cholesterol ( $\mathrm{CH}-\mathrm{T})$, high density cholesterol (HDL-Ch), and low-density cholesterol (LD-Ch).

Determination of body mass and body composition was performed by electrical impedance using the 220 InBody apparatus.

Venous blood samples were taken from all participants $(10 \mathrm{ml})$ to be assayed for the concentrations of $\mathrm{T}$, $\mathrm{GH}$, and IGF-1. IGF-1 concentration in the serum was determined by immunoradiometric (IRMA) methods using a diagnostic kit DSL-2800 Active IGF-1 (Diagnostic System Laboratories, Webster, Texas, USA). Determination of the concentration of $T$ was performed using the radioimmunoassay (RIA) analysis of blood serum using DSL-2100 assay. Biochemical analysis of the lipid and lipoprotein profile TAG, T-Ch, LDL-Ch, HDL-Ch was performed with an enzymatic method by a fully automated analyzer (Siemens Dimension). All biochemical assays were performed in duplicate with the ICC between 0.92 and 0.97 .

\section{Physical activity program}

The physical exercise program applied to all the participants included 4 training sessions per week, with 2 sessions directed at the improvemnt of anaerobic power (resistance exercise), while 2 consisted of aerobic endurance exercise.

Aerobic training was performed on a stationary cycle ergometer, starting with 30 minutes of continuous exercise at an intensity of $70-75 \%$ of maximum heart rate (HR max). Every two weeks, the work volume was increased by 5 minutes in order to reach 60 minutes in the last two weeks of the experiment. Strength training had a holistic aproach, involving all major muscle groups (the back, chest, abdomen, arms and lower limbs). For the first four weeks, exercises were performed in 3 sets of $8-12$ reps with the resistance equal to $60-70 \%$ of $1 \mathrm{RM}$ and $2 \mathrm{~min}$ rest periods between sets. During the experiment, the number of sets of each exercise increased from 3 to 4 sets in weeks $5-8$, and respectively to 5 sets in weeks $9-12$ for each exercise.

The research project was approved by the Ethics Committee for Scientific Research at the Academy of Physical Education in Katowice, Poland. 


\section{Statistical analysis}

The data were analyzed using the Statistica 9.1 software. The descriptive analyses consisted of the mean and standard deviation. For all measured variables, the estimated sphericity was verified according to the Mauchly's W test, and the Greenhouse-Geisser correction was used when necessary. Before using parametric tests, the assumption of normality was verified using the Kolmogorov-Smirnov test (Maszczyk et al., 2012). The comparison of analyzed values before and after the introduction of the experimental factor, was carried out with a two-way repeated measures ANOVA. When significant differences were found, Tukey HSD post-hoc tests were used. The effect size (eta-squared; $\eta^{2}$ ) of each test was calculated for all analyses. Effect size was classified according to Hopkins (Hopkins, 2010). Statistical significance was set at $p<0.05$.

\section{Results}

Table 1 presents pre- and post-intervention values of morphological variables under analysis. A two-way repeated measures ANOVA revealed a statistically significant effect of the intervention program on the following variables: T-Ch $\left(\eta^{2}=0.542\right)$, HDL-Ch $\left(\eta^{2}=0.522\right)$, LDL-Ch $\left(\eta^{2}=0.587\right), T\left(\eta^{2}=0.603\right)$, IGF-1 $\left(\eta^{2}=0.512\right)$, $\mathrm{GH}\left(\eta^{2}=0.621\right)$ in the exp. group. Tukey's HSD post-hoc test revealed a statistically significant decrease of T-Ch $(p=0.002)$ and LDL-Ch $(p=0.001)$, while other post-hock tests revealed statistically significant increases of HDL-Ch $(p=0.002), T(p=0.001)$, IGF-1 $(p=0.003)$ and GH $(p=0.001)$ in comparison to pre-intervention values.

The increase trend of $T\left(\eta^{2}=0.071\right)$ and $\mathrm{HDL}\left(\eta^{2}=0.057\right)$ was observed only in the contr. group. However, the effect of the intervention program was not statistically significant $(p=0.052, p=0.064$ respectively).

Table 1. The lipoprotein profile and hormone concentrations before and after supplementation with steroidal saponins with results of a two-way repeated measures ANOVA

\begin{tabular}{|c|c|c|c|c|c|}
\hline Variables & Group & $\begin{array}{l}\text { Before } \\
X \pm S D\end{array}$ & $\begin{array}{c}\text { After } \\
X \pm S D\end{array}$ & $\mathrm{~F}$ & $P$ \\
\hline \multirow{2}{*}{$\mathrm{T}-\mathrm{Ch}$ (mg/dl) } & $1 \exp$ & $189.64 \pm 28.98$ & $178.53 \pm 25.05$ & \multirow{2}{*}{15.417} & \multirow{2}{*}{0.003} \\
\hline & II cont & $196.42 \pm 26.85$ & $192.36 \pm 24.89$ & & \\
\hline \multirow{2}{*}{ HDL-Ch (mg/dl) } & I exp & $65.44 \pm 16.80$ & $70.01 \pm 14.50$ & \multirow{2}{*}{14.261} & \multirow{2}{*}{0.003} \\
\hline & II cont & $68.90 \pm 4.33$ & $65.57 \pm 4.30$ & & \\
\hline \multirow{2}{*}{ LDL-Ch (mg/dl) } & $1 \exp$ & $103.01 \pm 21.20$ & $98.64 \pm 11.31$ & \multirow{2}{*}{17.231} & \multirow{2}{*}{0.002} \\
\hline & II cont & $127.63 \pm 11.35$ & $128.78 \pm 8.75$ & & \\
\hline \multirow{2}{*}{$\mathrm{GH}(\mathrm{ng} / \mathrm{ml})$} & I exp & $0.47 \pm 0.15$ & $0.52 \pm 0.16$ & \multirow{2}{*}{23.261} & \multirow{2}{*}{0.001} \\
\hline & II cont & $0.58 \pm 0.14$ & $0.57 \pm 0.12$ & & \\
\hline \multirow{2}{*}{ IGF-1 (ng/ml) } & $1 \exp$ & $150.01 \pm 30.17$ & $180.17 \pm 27.38$ & \multirow{2}{*}{13.250} & \multirow{2}{*}{0.003} \\
\hline & II cont & $191.98 \pm 25.87$ & $191.57 \pm 23.85$ & & \\
\hline \multirow{2}{*}{$\mathrm{T}(\mathrm{nmol} / \mathrm{l})$} & $I \exp$ & $13.19 \pm 5.34$ & $14.74 \pm 4.06$ & \multirow{2}{*}{18.510} & \multirow{2}{*}{0.001} \\
\hline & II cont & $17.88 \pm 5.14$ & $17.64 \pm 4.01$ & & \\
\hline
\end{tabular}

Table 2 presents the pre- and post-intervention values of body composition and body mass variables. A twoway repeated measures ANOVA revealed a statistically significant effect of the intervention on BM $\left(\eta^{2}=0.644\right)$, FFM $\left(\eta^{2}=0.417\right)$, and TBF $\left(\eta^{2}=0.817\right)$ in the exp. group. Not significant post-intervention differences were observed in 
TBW $\left(\eta^{2}=0.094\right)$ value. Thus, FFM significantly increased while TBF and BM decreased in comparison to the preintervention levels ( $p=0.001, p=0.001$ and $p=0.003$, respectively).

Similarly, not significant post-intervention differences were observed in the contr. group. The increase of FFM $\left(\eta^{2}=0.058\right)$ and FAT $\left(\eta^{2}=0.044\right)$ values, and degrease of BM $\left(\eta^{2}=0.084\right)$ value $(p=0.061, p=0.071, p=0.581$ respectively), were observed only.

Tahle 2. Pre- to post-intervention changes in body composition and body mass, before and after the saponins treatment with results of a two-way repeated measures ANOVA

\begin{tabular}{|c|c|c|c|c|c|}
\hline Variables & Group & $\begin{array}{l}\text { Before } \\
X \pm S D\end{array}$ & $\begin{array}{c}\text { After } \\
X \pm S D\end{array}$ & $\mathrm{~F}$ & $P$ \\
\hline \multirow{2}{*}{$\mathrm{BM}(\mathrm{kg})$} & I exp & $89.17 \pm 8.62$ & $87.02 \pm 8.58$ & \multirow{2}{*}{19.551} & \multirow{2}{*}{0.011} \\
\hline & II cont & $99.54 \pm 7.24$ & $99.38 \pm 7.57$ & & \\
\hline \multirow{2}{*}{ TBF (kg) } & I exp & $20.22 \pm 7.57$ & $18.87 \pm 7.44$ & \multirow{2}{*}{69.921} & \multirow{2}{*}{0.011} \\
\hline & II cont & $20.82 \pm 5.86$ & $20.22 \pm 5.93$ & & \\
\hline \multirow{2}{*}{ FFM (kg) } & I exp & $70.42 \pm 6.18$ & $70.88 \pm 5.55$ & \multirow{2}{*}{9.591} & \multirow{2}{*}{0.026} \\
\hline & II cont & $85.84 \pm 8.78$ & $84.77 \pm 8.26$ & & \\
\hline \multirow{2}{*}{ TBW (kg) } & I exp & $51.75 \pm 4.49$ & $53.37 \pm 5.04$ & \multirow{2}{*}{1.243} & \multirow{2}{*}{0.772} \\
\hline & II cont & $62.74 \pm 6.16$ & $61.71 \pm 14.39$ & & \\
\hline
\end{tabular}

\section{Discussion and conclusions}

Ergogenic aids, such as dietery supplements, are essential elements of competitive sports, yet they can also improve metabolic efficiency in recreationally active subjects of all ages. Milasius, dadeliene, Skernevicius (2009), showed a significant positive effect of supplements containing TT on acid-base equilibrium after short-term, high intensity anaerobic exercise in competitive athletes. This effect can also be observed among middle aged and elderly subjects. In our research project, steroidal saponin supplementation in a group of middle-aged men caused a statistically significant increase in resting T, GH and IGF-1. The results of the study partially confirmed Brown's et al., reports (2001), which showed an significant effect of saponin supplementation on serum testosterone concentration in men aged 30-59 years. However, in Brown's et al., study (2001) the ergogenic effect was not caused only by TT, as the reserchers used a complex supplement called DION. Milasius et al. (2009) also observed an increase in blood T concentration, but only during the first 10 days of the experiment with TT supplementation. The test results obtained in the experiment conducted are contrary to Neychev, Mitev's research (2005), which showed no change in concentration of testosterone, androstenadiol and luteinizing hormone (LH) after supplementation with TT. The differences between the results of our experiment and those obtained by Neychev, Mitev (2005), may be the result of different dosages used, different duration of the experiment, and most importantly, different criteria for selecting subjects for the research. In most studies where no effect of saponins on T concentration was observed, young, physically active men with a physiologically high resting concentration of T were involved (Neychev, Mitev, 2005; Poprzecki, Zebrowska, Cholewa, Zając, Waskiewicz, 2005). In our study, the research group included middle-aged men with physiologically low levels of resting $T$, which seems to be a key factor in the effectiveness of such supplementation. An important finding of the study includes the fact that the TT supplementation also caused an increase in resting concentrations of GH and IGF-1 in the experimental group compared to the placebo group. 
Studies indicate that treatment with recombinant $\mathrm{GH}$ in elderly subjects significantly affects body composition (Poprzecki et al., 2005; Zając, Wilk, Socha, Maszczyk, Chycki, 2014). This effect is particularly important in case of increased concentrations of both GH and T (Blackman et al., 2002), which took place in the current study. Apart from the significant increase of GH concentration in this study, an increase in resting IGF-1 was also observed. Our research indicates significant increase in FFM and a decrease of BF with a concomitant increase in TBW. Similar results were obtained in a project by Poole et al. (Poole et al., 2010). Although statistically significant changes in the concentration of $\mathrm{T}$ were not shown in the Poole et al., study (2010), there was a trend for increased FFM $(p<0.001)$ and a decrease in BF $(p<0.001)$. Adverse changes in the lipid profile which increase the risk of coronary heart disease are typical for middle-aged men. Our study, in which men aged 45-60 were supplemented with steroidal saponins, revealed significant changes in the lipid and lipoprotein profile after 12 weeks of the experiment. A significant decrease of total cholesterol (T-Ch), an increase in high density cholesterol (HDL-Ch), a decrease in low density lipoprotein (LDL-Ch) and a reduction in plasma triglycerides (TAG) were observed. It may be assumed that TT supplementation is not necessarily directly responsible for changes in the lipid and lipoprotein profile. We hypothesize that it is the result of higher resting cocentrations of $\mathrm{T}$ and $\mathrm{GH}$, which was also observed in studies by Zając et al. (2014). It seems that both GH as well as T have a profound effect on the lipid and lipoprotein profile. Testosterone metabolism inhibits the uptake of triglycerides and lipoprotein lipase activity (Amore, 2005). Studies indicate that testosterone treatment reduces the levels of LDL-Ch (Hare et al., 2014), and exerts additional effects on lipids, depending on the dose and form of treatment. Munzer, Harman, Sorkin, Blackman (2009), also describe the impact of recombinant $\mathrm{GH}$ treatment on decreases in $\mathrm{LDL}-\mathrm{Ch}$, but not T-Ch. Combined treatment with $\mathrm{rGH}$ and T affects the decrease in T-Ch and LDL-Ch as well. Research conducted by Zając et al. (2014) on a similar group of middle-aged men indicate that injections of $\mathrm{T}$ and $\mathrm{GH}$ resulted in a decrease of $\mathrm{T}-\mathrm{Ch}$, an increase in HDL-Ch, a marked reduction in LDL-Ch. This justifies the assumption that it is the increase in the concentration of $T$ and $\mathrm{GH}$ which affects the changes in lipid profile and not the direct effect of TT supplementation. The increase in the concentration of resting $\mathrm{T}$ and $\mathrm{GH}$, stimulates changes in the lipid and lipoprotein profile in overweight subjects, which was also observed in the current study. The ergogenic effects of TT supplementation are not limited to incresed concentrations of $\mathrm{T}$ and $\mathrm{GH}$, what affects the lipid and lipoprotein profile, but also related to incresed physical activity. In this research, contrary to most studies in this area, the subjects involved in the study were physicaly active prior to the experiment, so regular exercise was a continuation of the current lifestyle and the changes in the lipid profile were mainly caused by the TT supplementation. The analyzed results indicate that treatment or supplementation of individual hormone deficiencies can be a major form of counteracting the aging process.

\section{Acknowledgements}

The author's research is funded by grants of the Ministry of Science and Higher Education of Poland (NRSA2 02552 and NRSA3 03953). 


\section{References}

Adimoelja, A.(2000). Phytochemicals and the breakthrough of traditional herbs in the management of sexual dysfunctions. Int $J$ Androl, $23(2), 82-84$.

Amore, M. (2005). Partial androgen deficiency and neuropsychiatric symptoms in aging men. J. Endocrinol Invest, 28, 49-54.

Antonio, J., Uelmen, J., Rodriguez, R., Earnest, C. (2010). The effects of Tribulus terrestris on body composition and exercise performance in resistance-trained males. Int. J. Sport Nutr. Metab, 10, 208-215.

Bird, S.P., Tarpenning, K.M., Marino, F.E. (2006). Effects of liquid carbohydrate/essential amino acid ingestion on acute hormonal response during a single bout of resistance exercise in untrained men. Nutrition, 22 (4), 367-375.

Blackman, M.R., Sorkin, J.D., Munzer, T., Bellantoni, M.F., Busby-Whitehead, J., Stevens, T.E., Jayme, J., O'Connor, K.G., Christmas, C., Tobin, J.D., Stewart, K.J., Cottrell, E., St. Clair, C., Pabst, K.M., Harman, S.M. (2002). Growth hormone and sex steroid administration in healthy aged women and men: a randomized controlled trial. JAMA, 288, 2282-2292.

Brown, G.A., Vukovich, M.D., Martini, E.R., Kohut, M.L., Franke, W.D., Jackson, D.A., King, D.S. (2001). Endocrine and lipid responses to chronic androstenediol-herbal supplementation in 30 to 58 year old men. J Am Coll Nutr, 20, 520-528.

De Combarieu, E., Fizzati, N., Lovati, M., Mercalli, E. (2003). Furostanol saponins from Tribulus terrestris. Fitoterapia, 74 (6), $583-591$.

Gauthaman, K., Mohamed Saleem, T.S., Ravi, V., Sita, P.S., Niranjali, D.S. (2008). Alcoholic Extract of Terminalia Arjuna Protects Rabbit Heart against Ischemic-Reperfusion Injury, Role of Antioxidant Enzymes and Heat Shock Protein. World Acad. Sci. Engineer. Technol, 18, 488-498.

Hare, D.J., Lei P., Ayton, S., Roberts, B.R., Grimm, R., George, J.L., Bishop, D., Beavis, A., Donovan, S.J., McColl, G., Volitakis, I., Masters, C.L., Adlard, P.A., Cherny, R.A., Bush, A.I., Finkelstein, D.I., Doble, P. (2014). An iron-dopamine index predicts risk of parkinsonian neurodegeneration in the substantia nigra pars compacta. Chem. Sci, 5, 2160-2169.

Hopkins, W.G. (2010). Linear Models and Effect Magnitudes for Research, Clinical and Practical Applications. Sportscience, $14,49-57$.

Huang, J.W., Tan Jiang, S.H., Zhu, D.Y. (2003).Terrestrinins A and B, two new steroid saponins from Tribulus terrestris. J Asian Nat Prod Res, 10, 285-290.

Milasius, K., Dadeliene, R., Skernevicius, J. (2009). The influence of the Tribulus terrestris extract on the parameters of the functional preparedness and athletes' organism homeostasis. Fiziol Zh, 55: 89-96.

Munzer, T., Harman, S.M., Sorkin, J.D., Blackman, M.R. (2009). Growth hormone and sex steroid effects on serum glucose, insulin, and lipid concentrations in healthy older women and men. J Clin Endocrinol Metab, 94 (10), 3833-3841.

Neychev, V.K., Mitev, V.I. (2005).The aphrodisiac herb Tribulus terrestris does not influence the androgen production in young men. J Ethnopharmacol, 101 (1-3), 319-323.

Poole, C., Bushey, B., Foster, C., Campbell, B., Willoughby, D., Kreider, R., Taylor, L., Wilborn, C. (2010). The effects of a commercially available botanical supplement on strength, body composition, power output, and hormonal profiles in resistance-trained males. J Int Soc. Sport Nutr, 7, 34.

Poprzecki, S., Zebrowska, A., Cholewa, J., Zając, A., Waskiewicz, Z. (2005). Ergogenic effects of Tribulus Terrestris supplementation in men. J Hum Kin, 13, 41-50.

Rogerson, S., Riches, C.J., Jennings, C. Weatherby, R.P., Meir, R.A., Marshall-Gradisnik, S.M. (2007). The effect of five weeks of tribulus terretris suplementation on muscle strength and body composition during preseason training in elite rugby league players. J Strength Cond Res, 21 (2), 348-353.

Zając, A., Czuba, M., Poprzecki, S., Waśkiewicz, Z., Cholewa, J., Pilch, J., Chycki, J. (2010). Effects of Growth Hormone Therapy and Physical Exercise on Anaerobic and Aerobic Power, Body Composition, Lipoprotein Profile in Middle Aged Men. J Hum Kin, 25, $67-76$.

Zając, A., Wilk, M., Socha, T., Maszczyk, A., Chycki, J. (2014). Effects of growth hormone and testosterone therapy on aerobic and anaerobic fitness, body composition and lipoprotein profile in middle-aged men. Ann Agric Environ Med, 21 (1), $175-171$.

Cite this article as:. Wilk, M., Michalczyk, M., Chycki, J., Maszczyk, A., Czuba, M., Roczniok, R., Gołaś, A., Zając, A. (2016). Endocrine Responses to Physical Training and Tribulus Terrestris Supplememtation in Middle-Age Men. Central European Journal of Sport Sciences and Medicine, 13 (1), 65-71. DOI: 10.18276/cej.2016.1-06. 
